\title{
Notes from Underground: are cognitive-enhancing drugs respecting their promises?
}

\author{
Frank Larøi ${ }^{1,2 *}$ and Andrea Raballo ${ }^{3,4,5}$ \\ 1 Cognitive Psychopathology Unit, University of Liège, Liège, Belgium \\ 2 Intercommunale de Soins Spécialisés de Liège (Mental Health Sector), Liège, Belgium \\ ${ }^{3}$ Danish National Research Foundation, Centre for Subjectivity Research, University of Copenhagen, Denmark \\ ${ }^{4}$ Department of Psychiatry, Hvidovre Hospital, University of Copenhagen, Hvidovre, Denmark \\ ${ }^{5}$ Department of Mental Health, AUSL di Reggio Emilia, Reggio Emilia, Italy
}

\section{Edited by:}

Salvatore Campanella, Université Libre de Bruxelles, Belgium

\section{Reviewed by:}

Michela Balconi, Catholic University of Milan, Italy

Philippe Detimary, Catholic University of Leuven, Belgium

\section{*Correspondence:}

Frank Larøi, Cognitive Psychopathology Unit, Department of Cognitive

Sciences, University of Liège, Bd. du Rectorat (B33), B-4000 Liège, Belgium. e-mail: flaroi@ulg.ac.be
Cognitive deficits are increasingly acknowledged as a highly relevant clinical feature of schizophrenia, susceptible of therapeutic intervention. Considerable efforts have been devoted to identify and develop pharmacological agents that provide cognitive-enhancing benefits to patients with schizophrenia. However, results from studies are not univocal and the "real world" cognitive functioning gain provided by these agents seems disappointingly modest. Three issues are crucial in this respect. First, there is no convincing evidence that atypical agents are any better than typical agents in improving cognitive functioning. Second, even though studies observe statistically significant treatment effects on cognition, these gains are poor or imperceptible on a clinical and functional level. Third, studies do not report differences in treatment effect compared to placebo effect in terms of their cognitive enhancing effects. Finally, examples of interventions that do provide important and meaningful improvements in cognitive functioning in schizophrenia patients will be briefly presented.

\section{INTRODUCTION}

Cognitive deficits are increasingly being acknowledged as an important feature of schizophrenia (Heinrichs, 2005) and, hence, as a potential therapeutic target. Moderate to severe cognitive impairment is frequently observed in schizophrenia both in early and chronic phases with a substantial impact on the global functional impairment and the long-term outcome (Kraus and Keefe, 2007). In this respect, a recent review of 16 longitudinal studies (Green and Nuechterlein, 2004) confirmed that cognition can reliably predict long-term outcome with medium to large effect sizes $(0.5-0.8$ or higher). Such findings spurred the United States National Institute of Mental Health to target cognitive deficits and boosted the search for ad hoc pharmacological intervention (cf. Marder and Fenton, 2004; Kraus and Keefe, 2007).

However, despite the considerable financial and research efforts that have been devoted to identify and develop pharmacological agents, there seems to be little evidence of significant cognitiveenhancing benefits for the current compounds. Indeed, results from Randomized Controlled Trial (RCT) studies are not univocal and the "real world" cognitive functioning gain provided by these agents seems disappointingly modest or, at least, of problematic "bench to bedside" translation.

Following this line, Green (2007) has remarked that we are becoming "less comfortable with pinning our hopes on antipsychotic medications as a way to achieve cognitive improvement" (p. 992). Quite on the contrary, alternative intervention strategies, such as cognitive/metacognitive-based ones, resulted more effective in empowering cognitive skills with long-lasting and meaningful improvements on social and professional functioning (cf. Wexler and Bell, 2005; Moritz and Woodward, 2007; Medalia and Choi,
2009). Thus, Harvey (2009) concludes a recent, comprehensive review of the literature in the following way: "Although potential pharmacological targets have been identified, previous results have been disappointing across multiple treatment targets. Optimism should arise from the findings that cognitive remediation interventions improve both cognitive performance and functional outcomes" (p. 333). This conclusion rings particularly important also in terms of strategic resource allocation, since the generalization of cognitive/metacognitive-based interventions at the level of mental health service delivery seems currently to be the most robust evidence-based option to target cognitive functioning.

Three further issues, which will be discussed below, are crucial in this respect. First, there is no convincing evidence that atypical agents are any better than typical agents in improving cognitive functioning. Second, even though studies observe statistically significant treatment effects on cognition, these gains are poor or imperceptible on a clinical and functional level. Third, studies do not report differences in treatment effect compared to placebo effect in terms of their cognitive enhancing effects.

\section{TYPICAL VERSUS ATYPICAL NEUROLEPTIC TREATMENT}

Systematic study of the effects of atypical (or second generation) neuroleptics on the neuropsychological deficits in schizophrenia began in the early 1990s with the idea that these medications might improve certain aspects of neurocognitive functioning, especially when compared with first generation (or typical) neuroleptics. However, studies do not seem to point to a particular drug or group of drugs that are more effective in enhancing cognitive impairment in schizophrenia patients and this, in particular, when comparing atypical and typical neuroleptics. Furthermore, Heinrichs (2007) 
concludes that cognitive deficits in patients with schizophrenia appear for the most part unresponsive to antipsychotic medication and this regardless of the type of drug. Mishara and Goldberg (2004) have shown that the presumed superiority of atypical neuroleptics in enhancing cognition was absent in a large patient sample when compared to typical neuroleptics. In a recent article summarizing the findings of the Clinical Antipsychotic Trials in Intervention Effectiveness (CATIE) study, the authors confirm that there were no significant differences between the first and second generation neuroleptics in terms of changes in cognitive domains (Keefe et al., 2007). Furthermore, as noted by Palmer et al. (2009), while overall changes in each of the groups were statistically significant, they were small and of questionable functional importance and (especially in the absence of an untreated group) could at least partially reflect practice effects. Moreover, upon further inspection, findings in this study suggest that the first generation agents actually revealed greater improvement compared to the second generation agents. Finally, a more recent trial also suggests no differential cognitive benefit of second generation versus first generation neuroleptics (Davidson et al., 2009). Thus, not only do studies fail to point to a particular drug or group of drugs that are more effective in enhancing cognitive impairment in schizophrenia but, in the light of the modest documented effect sizes, "there appears little reason to be very enthusiastic about the cognitive benefits of either class of medication" (Palmer et al., 2009, p. 376).

\section{TREATMENT EFFECT VERSUS LEARNING EFFECT}

In studies that have shown cognitive benefits, the effect sizes are often small and probably of little (or no) clinical significance. Heinrichs (2007) re-examined the treatment benefits (by comparing raw test scores at baseline and following 2 months) of the CATIE study (Keefe et al., 2007), revealing that patients' recall of a 12-noun word list improved, on average, by one-tenth of a word during this period. Further, word production on a verbal fluency task improved by an average of three-tenths of an additional word, and there were 1.5 fewer perseverative errors on the 64-card version of the Wisconsin Card Sorting test. Thus, even if studies reveal cognitive improvements, the benefits are small, and it is doubtful that they will have an impact on a clinical, social, or vocational level. What is more, these slight cognitive improvements may simply be attributed to a test-retest (learning) effect. Such a learning effect might magnify statistical differences in laboratory performances, but it is quite unlikely to offer any meaningful clinical proxy of daily life functioning. Finally, cognitive benefits were only evaluated after 2 months of treatment and thus the long-term benefits are not known. This unfortunately compromises the possibility of truly assessing the clinical impact in terms of enduring benefits.

\section{TREATMENT EFFECT VERSUS PLACEBO EFFECT}

When studies have included a placebo group, however, the findings are even more dramatic. Four previous double-blind, placebo-controlled trials (Friedman et al., 2002; Tugal et al., 2004; Freudenreich et al., 2005; Fagerlund et al., 2007) observed that change in cognitive functioning when given donepezil (a cholinesterase inhibitor) did not significantly differ from the change observed when given placebo. Similar negative results have been observed for other agents such as Modafinil (Freudenreich et al., 2009).
However, these studies involved few patients (no study enrolled more than 36 patients). More recently, a large $(N=250)$ doubleblind placebo-controlled trial on donepezil (Keefe et al., 2008) provided evidence of a larger placebo compared to treatment effect. When compared with the placebo treatment group, donepezil was not more effective for the improvement of cognitive impairment in schizophrenia patients. Furthermore, although both treatment groups experienced mild cognitive test performance improvement, the effect size of cognitive improvement was found to be markedly higher in the placebo group (0.45) compared to the donepezil group (0.26). Furthermore, the placebo group showed statistically greater improvement than the donepezil group at follow-up (12 weeks). This led the authors to suggest that, based on diminished practiceinduced learning relative to the placebo group, donepezil treatment might have exerted a negative influence on cognition.

\section{CONCLUSIONS}

Despite wide-spread optimism, available pharmacological agents do not seem to provide substantial cognitive-enhancing effects other than the symptomatic benefit on the major, conventional psychopathological dimensions of schizophrenia. In this respect, three core issues deserve critical consideration and which hopefully will also be addressed in future studies. First, despite the enormous resources used to develop atypical agents, there is no convincing evidence that these are any better than typical agents in improving cognitive functioning. Thus, although atypical agents may provide more favorable profiles in terms of compliance and overall clinical management, there is till now no robust evidence that these agents have a particular and appreciable advantage in terms of improving cognitive functioning. Second, even though studies (whether they are studying typical or atypical agents) find statistically significant treatment effects on cognition, these gains are poor or almost impalpable on a clinical and functional level. Furthermore, due to time constraints in the experimental designs, we seriously lack crucial information on the stability of the long-term effects on cognition. Future research needs to critically and specifically address the size and the temporal profile of the cognitive gains on a clinical and functional level. Third, and perhaps more fundamental, studies show that there is little difference (in terms of cognitive-enhancement) even compared to placebo: one study (Keefe et al., 2008) even showed a better cognitive improvement in the placebo group. Thus, for example, future (double-blind, placebo-controlled) studies will need to explore, in a more direct and critical manner, whether or not certain agents are actually having a negative effect on cognitive functioning in patients with schizophrenia.

For the time being, available pharmacological agents seem to provide modest (if any) appreciable cognitive benefits to patients suffering from schizophrenia. Furthermore, there seems to be rather scarce evidence of any direct specific cognitive-enhancing effect other than those secondary to a global deflation of the severity of psychopathology. In this respect, it seems reasonable to hypothesize that more resources - both financial and human - might be better off invested in complementary treatment schemes (e.g., cognitive remediation, vocational training, metacognitive training) that do provide important, direct, and meaningful improvements for patients. Indeed, a number of approaches to remediating cognition in schizophrenia have been developed and studied in the last 15 years and this literature has been reviewed in six meta-analytic 
studies. With the exception of one (Pilling et al., 2002), all have found moderate to large effect sizes (Kurtz et al., 2001; Suslow et al. 2001; Krabbendam and Aleman, 2003; Twamley et al. 2003; McGurk et al. 2007) - effect sizes far greater than those observed for any drug treatment. Also, these remediation effects are durable up to at least 6 months after the interventions are withdrawn. Finally, and most importantly, these neuropsychological gains translate to improvements in real-world activities (Larøi and Van der Linden, 2007; Levaux et al., 2009; Medalia and Choi, 2009). Similarly, cognitive remediation programmes address prominent, complementary functional domains such as social cognition - and studies have furthermore observed beneficial effects on functional outcome measures in patients post-treatment (for reviews see Couture et al.,

\section{REFERENCES}

Couture, S. M., Penn, D. L., and Roberts, D. L. (2006). The functional significance of social cognition in schizophrenia: a review. Schizophr. Bull. 32(Suppl. 1), 44-63.

Davidson, M., Galderisi, S., Weiser, M., Werbeloff, N., Fleischhacker, W. W., Keefe, R. S., Boter, H., Keet, I. P., Prelipceanu, D., Rybakowski, J. K., Libiger, J., Hummer, M., Dollfus, S., López-Ibor, J. J., Hranov, L. G., Gaebel, W., Peuskens, J., Lindefors, N., RiecherRössler, A., and Kahn, R. S. (2009). Cognitive effects of antipsychotic drugs in first-episode schizophrenia and schizophreniform disorder: a randomized, open-label clinical trial (EUFEST).Am. J. Psychiatry 166, 675-682.

Fagerlund, B., Søholm, B., Fink-Jensen, A. Lublin, H., and Glenthøj, B. Y. (2007). Effects of donepezil adjunctive treatment to ziprasidone on cognitive deficits in schizophrenia: a doubleblind, placebo-controlled study. Clin. Neuropharmacol. 30, 3-12.

Freudenreich, O., Henderson, D. C. Macklin, E. A., Evins, A. E., Fan, X., Cather, C., Walsh, J. P., and Goff, D. C. (2009).Modafinil for clozapine-treated schizophrenia patients: a double-blind, placebo-controlled pilot trial. J. Clin. Psychiatry 70, 1674-1680.

Freudenreich, O., Herz, L., Deckersbach, T., Evins, A. E., Henderson, D. C., Cather, C., and Goff, D. C. (2005). Added donepezil for stable schizophrenia: a double-blind, placebocontrolled trial. Psychopharmacology 181, 358-363.

Friedman, J. I., Adler, D. N., Howanitz, E., Harvey, P. D., Brenner, G., Temporini, H., White, L., Parrella, M., and Davis, K. L. (2002). A double blind placebo controlled trial of donepezil adjunctive treatment to risperidone for the cognitive impairment of schizophrenia. Biol. Psychiatry 51, 349-357.

Green, M. F. (2007). Cognition, drug treatment, and functional outcome in schizophrenia: a tale of two transitions. Am. J. Psychiatry 164, 992-994.
Green, M. F., and Nuechterlein, K. H. (2004). The MATRICS initiative: developing a consensus cognitive battery for clinical trials. Schizophr. Res. 72, 1-3.

Harvey, P. D. (2009). Pharmacological cognitive enhancement in schizophrenia. Neuropsychol. Rev. 19, 324-335.

Heinrichs, R. W. (2005). The primacy of cognition in schizophrenia. Am. Psychol. 60, 229-242.

Heinrichs, R. W. (2007). Cognitive improvement in response to antipsychotic drugs: neurocognitive effects of antipsychotic medications in patients with chronic schizophrenia in the CATIE trial. Arch. Gen. Psychiatry 64, 631-632.

Keefe, R. S. E., Bilder, R. M., Davis, S. M. Harvey, P. D., Palmer, B. W., Gold, J. M., Meltzer, H. Y., Green, M. F., Capuano, G., Stroup, T. S., McEvoy, J. P., Swartz, M. S., Rosenheck, R. A., Perkins, D. O., Davis, C. E., Hsiao, J. K., and Lieberman, J. A. (2007). Neurocognitive effects of antipsychotic medications in patients with chronic schizophrenia in the CATIE trial. Arch. Gen. Psychiatry 64, 633-647.

Keefe, R. S. E., Malhotra, A. K., Meltzer, H. Y., Kane, J. M., Buchanan, R. W., Murthy, A., Sovel, M., Li, C., and Goldman, R. (2008). Efficacy and safety of donepezil in patients with schizophrenia or schizoaffective disorder: significant placebo/practice effects in a 12-week, randomized, double-blind, placebo-controlled trial. Neuropsychopharmacology 33, 1217-1228.

Krabbendam, L., and Aleman, A. (2003). Cognitive rehabilitation in schizophrenia: a quantitative analysis of controlled studies. Psychopharmacology 169, 376-382.

Kraus, M. S., and Keefe, R. S. E. (2007). Cognition as an outcome measure in schizophrenia. Br. J. Psychiatry 191, 46-51.

Kurtz, M.M., Moberg, P. J., Gur, R. C., and Gur, R. E. (2001). Approaches to cognitive remediation of neuropsychological deficits in schizophrenia: a review

2006; Penn et al., 2008). At present, however, social cognition has not been shown to be amenable to enhancement via antipsychotic medication (Penn et al., 2008).

Needless to say, these two approaches to treatment and rehabilitation (namely pharmacotherapy and cognitive-based intervention strategies) are highly complementary and are usually combined in clinical reality. However, in order to maximize the benefits for patients, it is important to optimize the balance between these two intervention strategies according to the specific domains that require therapeutic empowerment. Future studies should furthermore address the combination of cognitive and pharmacological treatment schemes that provide the widest and more enduring benefits for the patients.

and meta-analysis. Neuropsychol. Rev. $11,197-210$.

Larøi, F., and Van der Linden, M. (2007). Revalidation cognitive et schizophrénie: pour une approche individualisée et centrée sur les activités de la vie quotidienne. Lett. Psychiatre 3, 50-53.

Levaux, M.-N., Vezzaro, J., Larøi, F., Van der Linden, M., Offerlin-Meyer, I., and Danion, J.-M. (2009). Cognitive remediation of the updating subcomponent of working memory in schizophrenia: a single-case study. Neuropsychol. Rehabil. 19, 244-273.

Marder, S. R., and Fenton, W. (2004). Measurement and treatment research to improve cognition in schizophrenia: NIMH MATRICS initiative to support the development of agents for improving cognition in schizophrenia. Schizophr. Res. 72, 5-9.

McGurk, S. R., Twamley, E. W., Sitzer, D. I., McHugo, G. J., and Mueser, K. T. (2007). A meta-analysis of cognitive remediation in schizophrenia. Am. J. Psychiatry 164, 1791-1802.

Medalia, A., and Choi, J. (2009). Cognitive remediation in schizophrenia. Neuropsychol. Rev. 19, 353-364.

Mishara, A. L., and Goldberg, T.E. (2004) A meta-analysis and critical review of the effects of conventional neuroleptic treatment on cognition in schizophrenia: opening a closed book. Biol. Psychiatry 55, 1013-1022.

Moritz, S., and Woodward, T. S. (2007) Metacognitive training in schizophrenia: from basic research to knowledge translation and intervention. Curr. Opin. Psychiatry 20, 619-625.

Palmer, B. W., Dawes, S. E., and Heaton, R. K. (2009). What do we know about neuropsychological aspects of schizophrenia? Neuropsychol. Rev. 19 365-384.

Penn, D. L., Sanna, L. J., and Roberts, D. L. (2008). Social cognition in schizophrenia: an overview. Schizophr. Bull. 34, 408-411.

Pilling, S., Bebbington, P., Kuipers, E., Garety, P., Geddes, J., Martindale, B.
Orbach, G., and Morgan, C. (2002). Psychological treatments in schizophrenia: II. Meta-analyses of randomized controlled trials of social skills training and cognitive remediation. Psychol. Med. 32, 783-791.

Suslow, T., Schonauer, K., and Arolt, V. (2001). Attention training in the cognitive rehabilitation of schizophrenic patients: a review of efficacy studies. Acta Psychiatr. Scand. 103, 15-23.

Tugal, Ö., Yazici, K. M., Yagcioglu, E. A., and Gögüs, A. (2004). A double-blind, placebo controlled, cross-over trial of adjunctive donepezil for cognitive impairment in schizophrenia. Int. J. Neuropsychopharmacol. 7, 117-123.

Twamley, E. W., Jeste, D.V., and Bellack, A. S. (2003). A review of cognitive training in schizophrenia. Schizophr. Bull. 29, 359-382.

Wexler, B. E., and Bell, M. D. (2005). Cognitive remediation and vocational rehabilitation for schizophrenia. Schizophr. Bull. 31, 931-941.

Conflict of Interest Statement: The authors declare that the research was conducted in the absence of any commercial or financial relationships that could be construed as a potential conflict of interest.

Received: 26 April 2010; paper pending published: 17 May 2010; accepted: 07 September 2010; published online: 30 September 2010.

Citation: Larøi F and Raballo A (2010) Notes from Underground: are cognitiveenhancing drugs respecting their promises? Front. Psychology 1:158. doi: 10.3389/ fpsyg.2010.00158

This article was submitted to Frontiers in Psychopathology, a specialty of Frontiers in Psychology.

Copyright (C) 2010 Larøi and Raballo. This is an open-access article subject to an exclusive license agreement between the authors and the Frontiers Research Foundation, which permits unrestricted use, distribution, and reproduction in any medium, provided the original authors and source are credited. 